n.tronos

glyndŵn

Glyndŵr University

Glyndŵr University Research Online

Computing

Computer Science

4-1-2008

\title{
Autofocus for ISAR Imaging Using Higher Order Statistics
}

\author{
Zhishun She \\ Glyndwr University, z.she@glyndwr.ac.uk
}

Y Liu

Follow this and additional works at: http://epubs.glyndwr.ac.uk/cair

Part of the Computer Engineering Commons

\section{Recommended Citation}

She, Z. \& Liu, Y. (2008) 'Autofocus for ISAR Imaging Using Higher Order Statistics'. IEEE Geoscience and Remote Sensing Letters, 5(2), 299-303

This Article is brought to you for free and open access by the Computer Science at Glyndŵr University Research Online. It has been accepted for inclusion in Computing by an authorized administrator of Glyndŵr University Research Online. For more information, please contact

d.jepson@glyndwr.ac.uk. 


\title{
Autofocus for ISAR Imaging Using Higher Order Statistics
}

\begin{abstract}
Autofocus is imperative for inverse synthetic aperture radar (ISAR) imaging. In this letter, a new approach for ISAR autofocus is developed by using fourth-order statistics properties of the radar's return signal. After the ISAR signal model is established, the approach is described. The results of processing real data confirm the effectiveness of the proposed approach and show its capability for suppressing noise. The developed approach has a numerical stability and a smaller computational load compared with the maximum image contrast and the minimum image entropy methods.
\end{abstract}

\section{Keywords}

Array calibration, autofocus, higher order statistics (HOS), inverse synthetic aperture radar (ISAR)

\section{Disciplines}

Computer Engineering

\section{Comments}

Copyright (C) 2008 IEEE. Reprinted from IEEE Geoscience and Remote Sensing Letters 2008, 5(2), 299-303 This material is posted here with permission of the IEEE. Such permission of the IEEE does not in any way imply IEEE endorsement of any of the products or services of Glyndŵr University Wrexham. Internal or personal use of this material is permitted. However, permission to reprint/republish this material for advertising or promotional purposes or for creating new collective works for resale or redistribution must be obtained from the IEEE by writing to pubs-permissions@ieee.org. By choosing to view this document, you agree to all provisions of the copyright laws protecting it. The published article is also available on the IEEE website http://ieeexplore.ieee.org 


\title{
Autofocus for ISAR Imaging Using Higher Order Statistics
}

\author{
Zhishun She and Y. Liu
}

\begin{abstract}
Autofocus is imperative for inverse synthetic aperture radar (ISAR) imaging. In this letter, a new approach for ISAR autofocus is developed by using fourth-order statistics properties of the radar's return signal. After the ISAR signal model is established, the approach is described. The results of processing real data confirm the effectiveness of the proposed approach and show its capability for suppressing noise. The developed approach has a numerical stability and a smaller computational load compared with the maximum image contrast and the minimum image entropy methods.
\end{abstract}

Index Terms-Array calibration, autofocus, higher order statistics (HOS), inverse synthetic aperture radar (ISAR).

\section{INTRODUCTION}

$\mathbf{S}$ INCE its origins in the 1950s, synthetic aperture radar (SAR) has been extensively developed and widely exploited for civilian applications and surveillance purposes. SAR is typically carried on a moving platform such as an aircraft or a satellite that is intended to be used in air-to-ground imaging of terrain [1]. Inverse SAR (ISAR) [2] is usually used for imaging of moving targets. ISAR signal processing usually includes motion compensation and image formation. Motion compensation involves the removal of the translational motion between the object and the radar prior to image formation. However, motion compensation in ISAR is more challenging than SAR because ISAR objects are often not cooperative.

ISAR imaging is a coherent process and requires that the errors in synthesizing the azimuthal aperture are less than a fraction of the radar wavelength. To satisfy this strict requirement, motion compensation in ISAR is usually carried out in two steps. The first step is range realignment in which the high resolution range profiles are aligned in the range direction by placing the returns of different pulses from the same scatterer in the same range cell. It is a coarse compensation of translational motion. The second step is autofocus, which removes the residual translational motion. It applies a phase correction to the range-aligned signals in the azimuthal direction. Autofocus is the fine compensation of translational motion.

ISAR autofocus has been a fascinating area of research in the last two decades, and many methods for ISAR autofocus have been proposed. A simple approach to ISAR autofocus is

Manuscript received December 13, 2007. This work was supported by the Natural Science Foundation Project of CQ CSTC.

The authors are with the School of Science and Technology, North East Wales Institute of Higher Education (NEWI), University of Wales, Wrexham, LL11 2AW, U.K. (e-mail: z.she@newi.ac.uk).

Digital Object Identifier 10.1109/LGRS.2008.916074 to find a range cell containing a strong scatterer, which is the dominant scatterer algorithm (DSA) [3]. For a complex target that does not have a stable prominent scatterer, a phase gradient is estimated by averaging the phase differences over each range cell, and then an iterative phase correction is conducted, which is called phase gradient autofocus (PGA) [4]. Recently, methods based on image contrast [5] and entropy [6] have been proposed for ISAR autofocus. They are the parametric methods and use a polynomial model to approximate the translational motion [12], [13]. In these approaches, many images are produced with different polynomial coefficients. One that produces the maximum image contrast or the minimum image entropy is selected as the optimal focusing parameter. However, the computational cost is high.

ISAR autofocus can be considered as a problem of sensor array processing and be solved from the perspective of array calibration. Algorithms for ISAR autofocusing were developed based on second-order statistics (SOS) of synthetic array data [7], [9]. The bispectrum (third-order statistics) was used for cross-range spotlight SAR autofocus [11]. In this letter, a new method for ISAR autofocus using a fourth-order statistic is proposed. It is a nonparametric approach and has a numerical stability and low computational load. Its advantage is that the additive Gaussian noise can be suppressed in the fourth-order cumulant domain [8], which results in an enhanced signal-tonoise ratio (SNR) and the low threshold of SNR to produce good quality ISAR images. This unique characteristic constitutes the motivation of work conducted in this study. This letter is organized as follows. A mathematical model of the returned ISAR signal is given in Section II; Section III describes the autofocus algorithm in detail; processing of experimental ISAR data, computational complexity, noise suppression, and image quality are discussed in Section IV.

\section{ISAR SIGNAL MODEL}

Suppose that a moving object is flying in a straight line $o x$ as shown in Fig. 1. The motion of a rigid object can be decomposed into two parts: a translational motion of a certain reference point $o$ on the object and a rotational motion of the object about the point $o$. Let the Cartesian coordinate xoy be fixed on the object with range along the $y$-axis and cross-range along the $x$-axis when the object is at a broadside position to the radar. The radar transmits $M$ stepped-frequency bursts. The aspect angle of the object relative to the radar-line-of-sight (RLOS) and the distance from the radar to the point $o$ when the $m$ th burst is sent are represented by $\theta_{m}$ and $R_{o m}$, respectively, where $m=0, \ldots, M-1$. 


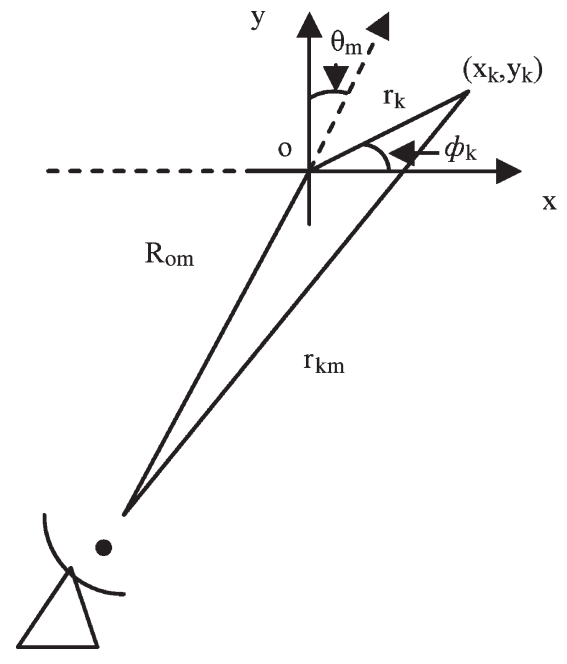

Fig. 1. ISAR imaging geometry.

Assume that there are $K$ scatterers on the object. The $k$ th scatterer is situated a distance $r_{k m}$ from the radar when the $m$ th burst is sent. The range between the radar and the $k$ th scatterer with coordinate $\left(r_{k}, \phi_{k}\right)$ or $\left(x_{k}, y_{k}\right)$ is given by

$$
r_{k m}=\left[R_{o m}^{2}+r_{k}^{2}+2 R_{o m} r_{k} \sin \left(\theta_{m}+\phi_{k}\right)\right]^{\frac{1}{2}} .
$$

If the distance to the object is much larger than the size of the object, that is, $R_{o m} \gg r_{k}$, we have the approximation

$$
r_{k m} \cong R_{o m}+x_{k} \sin \theta_{m}+y_{k} \cos \theta_{m} .
$$

Let $\rho_{k}$ denote the complex reflected signal of the $k$ th scatterer, which is assumed to be independent of the illuminating frequency and the aspect angle. For each burst, $L$-stepped frequencies $f_{l}=f_{0}+l \Delta f l=0, \ldots, L-1$ are used where $f_{0}$ and $\Delta f$ are the initial and step frequency, respectively. The received signal $S_{k l m}$ resulting from the $k$ th scatterer and the $l$ th illuminating frequency during the $m$ th burst can be written as

$$
S_{k l m}=\rho_{k} \exp \left\{-j 4 \pi f_{l} r_{k m} / c\right\} .
$$

The total returned signal $s_{l m}$ caused by the lth illuminating frequency of the $m$ th burst is

$$
s_{l m}=\sum_{k=1}^{K} \rho_{k} \exp \left\{-j 4 \pi f_{l} r_{k m} / c\right\} .
$$

After the pulse compression in the range direction using an inverse discrete Fourier transform (IDFT), the complex envelope in the $n$th range cell of the $m$ th burst becomes [9]

$$
z_{n m}=\exp \left\{-j 4 \pi f_{0} R_{o m} / c\right\} e_{n m}+w_{n m}
$$

where $e_{n m}, m=0, \ldots, M-1, n=0, \ldots, N-1$ is the returned signal resulting from the rotational motion of the object, $w_{n m}$ is the complex envelope of additive noise, and $N$ is the total number of range cells. It is assumed that $w_{n m}$ are independent identically distributed complex Gaussian noise components with zero mean and variance $\sigma_{w}^{2}$, because ISAR usually conducts ground-to-air imaging.

Following the range compression, range realignment is done to align the high-resolution range profiles in the range direction so that the returns of different pulses from the same scatterer lie in the same range cell. After range realignment is accomplished, the signal model $z_{n m}$ for ISAR autofocus is written as [9]

$$
z_{n m}=e_{n 0} d_{m}+w_{n m}
$$

where $e_{n 0}, n=0, \ldots, N-1$ is the return signal of the first burst caused by the rotational motion of the object, and $d_{m}=$ $\exp \left\{-j 4 \pi R_{o m} / \lambda\right\}$, which is the complex signal that ISAR autofocus needs to estimate, and $\lambda$ is the wavelength corresponding to $f_{0}$. The unique property of the proposed approach is the nonparametric estimation of the focusing point distance, $R_{o m}$. The complex exponential signal vector whose phase corresponds to the translational motion rather than the phase itself is estimated.

There are two assumptions made for the signal model in (6) [9]. One is that the target center of rotation is stationary within a burst. The other is that the motion of the target's center is limited to moving along a straight line, and the target's center of rotation will not change position, although the target may exhibit the rotation motion. These two assumptions can be satisfied by increasing the pulse repetition frequency (PRF) appropriately.

\section{Autofocusing Using Higher Order Statistics}

A fourth-order cumulant is used to measure the higher order statistics (HOS) of ISAR signals. Although the different cumulants for a complex variable exist [8], a simple scheme, a diagonal slice, is chosen, which is defined as

$$
C_{4, z_{n}}\left(i, j^{*}, j, j^{*}\right)=\operatorname{Cum}\left(z_{n i}, z_{n j}^{*}, z_{n j}, z_{n j}^{*}\right)
$$

where * denotes conjugation and Cum represents the fourthorder cumulant. Substituting (6) into (7), we obtain

$$
\begin{aligned}
& C_{4, z_{n}}\left(i, j^{*}, j, j^{*}\right)= \\
& \operatorname{Cum}\left(e_{n 0} d_{i}+w_{n i}, e_{n 0}^{*} d_{j}^{*}+w_{n j}^{*}, e_{n 0} d_{j}+w_{n j}, e_{n 0}^{*} d_{j}^{*}+w_{n j}^{*}\right) .
\end{aligned}
$$

Because the additive noise $w_{n m}$ is independent of the returned signal $e_{n 0} d_{m}$ in (6), we have

$$
\begin{aligned}
C_{4, z_{n}}\left(i, j^{*}, j, j^{*}\right)=\operatorname{Cum} & \left(e_{n 0} d_{i}, e_{n 0}^{*} d_{j}^{*}, e_{n 0} d_{j}, e_{n 0}^{*} d_{j}^{*}\right) \\
+ & +\operatorname{Cum}\left(w_{n i}, w_{n j}^{*}, w_{n j}, w_{n j}^{*}\right) .
\end{aligned}
$$

If the additive noise is Gaussian, the fourth-order cumulant of Gaussian noise is zero [10], and (9) is simplified into

$$
C_{4, z_{n}}\left(i, j^{*}, j, j^{*}\right)=d_{i} d_{j}^{*} d_{j} d_{j}^{*} k_{n}=d_{i} d_{j}^{*} k_{n}
$$




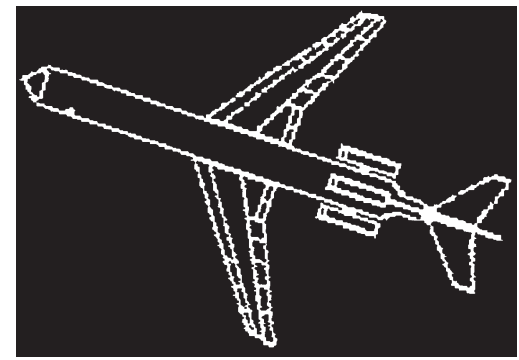

Fig. 2. Boeing 727 plan view.

where $k_{n}=\operatorname{Cum}\left(e_{n 0}, e_{n 0}^{*}, e_{n 0}, e_{n 0}^{*}\right)$ is the kurtosis of the signals in the $n$th range cell. The two-dimensional matrix format of (10) is

$$
C_{4, z_{n}}=k_{n} D D^{H}
$$

where $H$ denotes the Hermitian transpose and $D=\left[d_{0}, \ldots\right.$, $\left.d_{M-1}\right]^{T}$, which is the complex vector that ISAR autofocus needs to estimate. Equation (11) shows that the rank of the diagonal slice matrix is one because the number of linearly independent column vectors in the matrix is one. Therefore the eigenvector corresponding to maximum eigenvalue of the diagonal slice matrix is proportional to $D$ and can be used for ISAR autofocus.

The foregoing derivations are based on the assumption that the exact fourth-order cumulants are available. However, in practice these must be estimated from a finite number of data samples. Because vector $D$ is independent of the range cell index, the sample cumulant matrix can be estimated by replacing the ensemble average with one over range cells. The estimator is expressed by

$$
\hat{C}_{4, z_{n}}\left(i, j^{*}, j, j^{*}\right)=\frac{1}{N} \sum_{n=0}^{N-1}\left|z_{n j}\right|^{2} z_{n i} z_{n j}^{*} .
$$

In summary, a new ISAR imaging algorithm using HOS includes five steps: First, the returned signal is compressed in the range direction. Second, the compressed range profiles are aligned by correlation processing [2]. Third, the fourthorder cumulant matrix is estimated by averaging over range cells. Fourth, the eigenvector corresponding to the maximum eigenvalue of the diagonal slice matrix is used for autofocus. It is chosen as the estimation of a complex exponential signal vector resulting from the translational motion, and the $m$ th range aligned profile is multiplied by the conjugation of this vector. Finally, the ISAR image is formed by cross-range compression.

\section{EXPERIMENTAL RESULTS}

Thanks to Prof. B.D. Steinberg of the University of Pennsylvania, we received the experimental data of a Boeing 727 whose top view is shown in Fig. 2. The commercial Boeing 727 aircraft was flying into Philadelphia International Airport. The central frequency of radar was $9.6 \mathrm{GHz}$ (X-band) $(\lambda=3.123 \mathrm{~cm})$. Range resolution of $1 \mathrm{~m}$ was achieved by transmitting a narrow pulse $7 \mathrm{~ns}$ wide. Signals in 120 range cells were recorded, and the PRF was $400 \mathrm{~Hz}$. Because the target

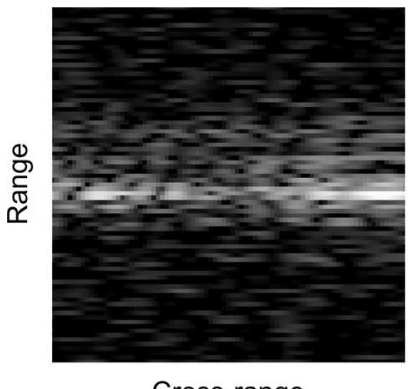

Cross-range

(a)

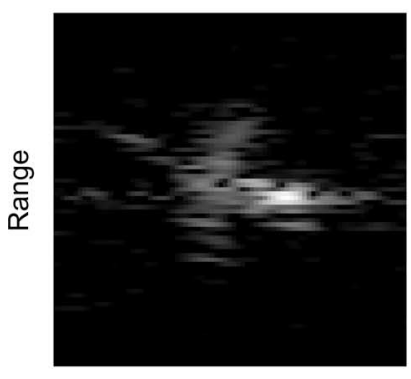

Cross-range

(c)

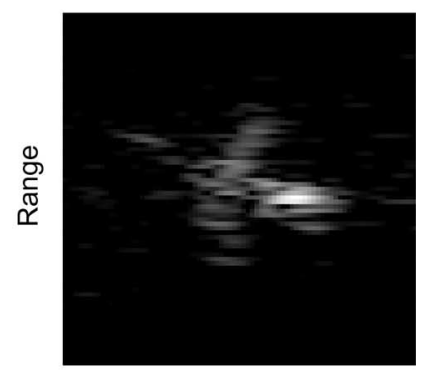

Cross-range

(b)

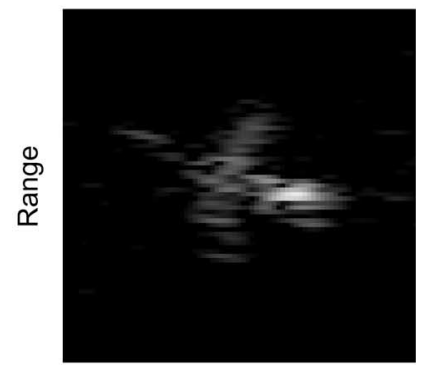

Cross-range

(d)

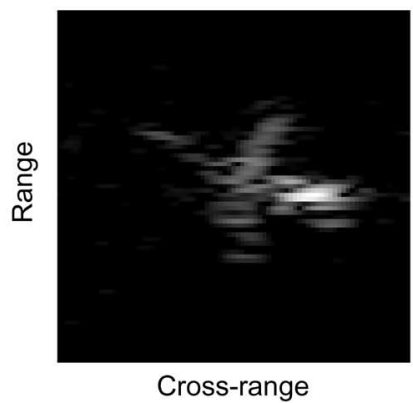

(e)

Fig. 3. ISAR images of a real Boeing 727 aircraft. (a) Unfocused, (b) focused by HOS, (c) focused by SOS, (d) focused by maximum image contrast, and (e) focused by minimum image entropy.

was at the broadside position, the radial velocity of the target with respect to the radar was small, and the first assumption described in Section II could be satisfied.

After the range compression, 32 range profiles were chosen. The change in aspect angle of the target was $0.4^{\circ}$, and the sampling interval of the aspect angle was $2.18 \times 10^{-4} \mathrm{rad}$, which met the requirement on sampling interval for the second assumption discussed in Section II [9]. The real data were first processed with range realignment. Then the estimated diagonal slice matrix was computed by averaging over 120 range cells using (12), which gave a $32 \times 32$ matrix. Next, the eigenvector corresponding to the maximum eigenvalue of the estimated matrix was used for autofocus. Finally, the focused image was obtained by cross-range processing. The ISAR image of the Boeing 727 focused with the fourth-order culumant is shown in Fig. 3(b) where the SNR is about $10 \mathrm{~dB}$. SNR is defined as $\mathrm{SNR}=10 \log _{10}\left(P_{s} / P_{n}\right)$ where $P_{s}$ and $P_{n}$ are the powers of the target and the noise, respectively. They are estimated by the signal powers in the range cells with and without the target, respectively. Compared with the unfocused ISAR image presented in Fig. 3(a), it shows the effectiveness of the proposed 


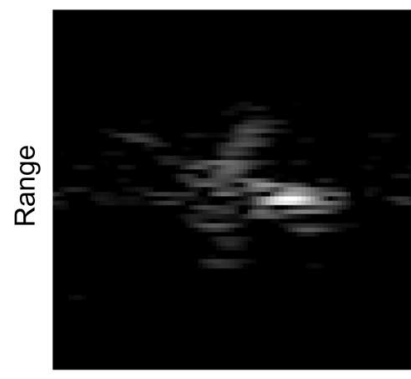

Cross-range

(a)

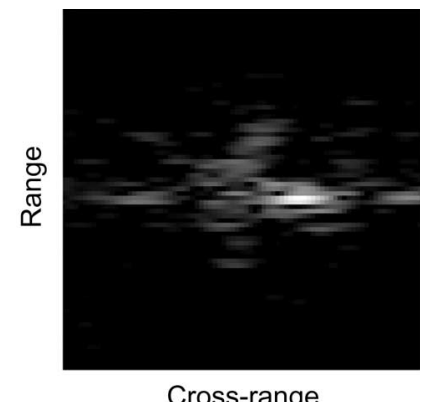

(b)
Fig. 4. ISAR images at SNR $=-10 \mathrm{~dB}$. (a) Focused by HOS, and (b) focused by SOS.

method. The focused ISAR images using the SOS (the signal subspace approach), the maximum image contrast, and the minimum image entropy are shown in Fig. 3(c)-(e), respectively. The focused images are quite impressive compared with the plan view of Boeing 727 as shown in Fig. 2.

The computational loads of SOS, HOS, maximum image contrast, and minimum image entropy for ISAR autofocus can be compared in the terms of the number of multiplication as follows: $M N+M^{2} N+4 M^{3} / 3, M N+3 M^{2} N+$ $4 M^{3} / 3, L_{1}\left(2 M N+M N / 2 \log _{2} M\right)+M N$ and $L_{2}(3 M N+$ $\left.M N / 2 \log _{2} M\right)+M N$, respectively, where $L_{1}$ and $L_{2}$ are the numbers of iteration for the optimization. In the experiment we had $M=32, N=120, L_{1}=71$, and $L_{2}=82$. The numbers of multiplication for SOS, HOS, maximum image contrast, and minimum image entropy are $1.70 \times 10^{5}, 4.16 \times 10^{5}, 1.23 \times$ $10^{6}$, and $1.74 \times 10^{6}$, respectively. Because maximum image contrast and minimum image entropy include the optimization procedures, their computational loads are larger than those of the SOS and HOS approaches. The HOS has a slightly greater computational load than the SOS. For the same number of iterations, the maximum image contrast method is more efficient in computational complexity than the minimum image entropy method.

To investigate the capability to suppress noise, spatially Gaussian noise was added to the real data before autofocus. When the noise is added to get $\mathrm{SNR}=-10 \mathrm{~dB}$, the ISAR images focused by HOS and SOS (the signal subspace approach) methods [9] are shown in Fig. 4(a) and (b), respectively, indicating that the HOS approach is more robust to Gaussian noise than the SOS method.

To measure this enhancement statistically, ISAR autofocus was conducted 100 times using real data with added noise at each specified SNR level. The normalized image contrast was used to measure the focused quality [5]. The mean of the normalized image contrast is calculated by averaging the 100 normalized image contrasts at different SNR. The mean of ISAR image contrast focused by the HOS and SOS methods is shown in Fig. 5. It indicates that both provide the same focused quality if SNR is greater than $-5 \mathrm{~dB}$. However, as SNR decreases, the HOS approach degrades more slowly than the SOS method. The SNR thresholds of $90 \%$ focused image contrast for the HOS and SOS methods are -18 and $-8 \mathrm{~dB}$, respectively.

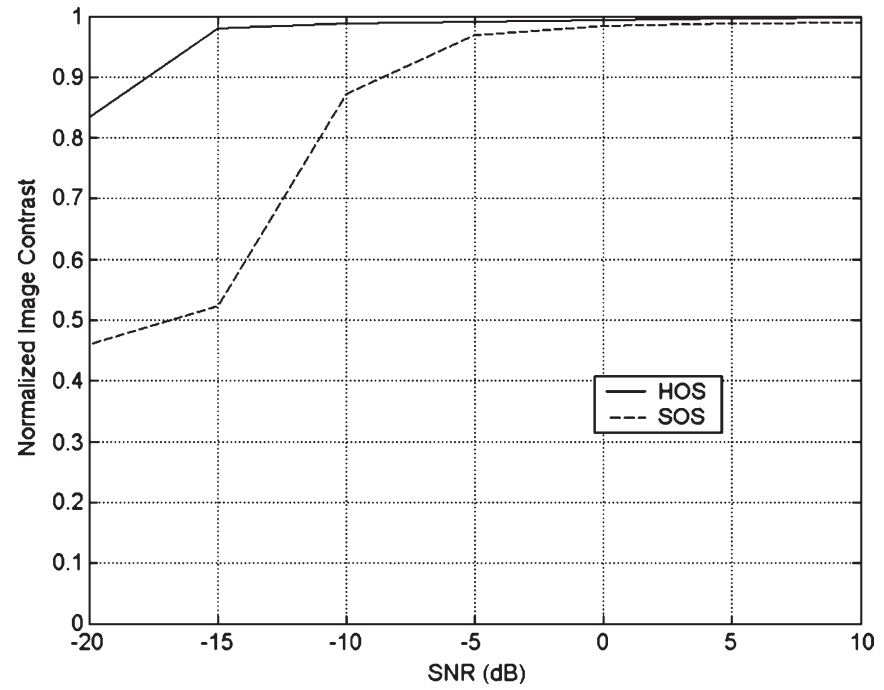

Fig. 5. Mean of ISAR image contrast versus SNR.

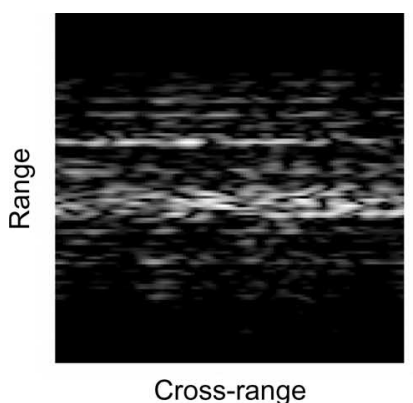

(a)

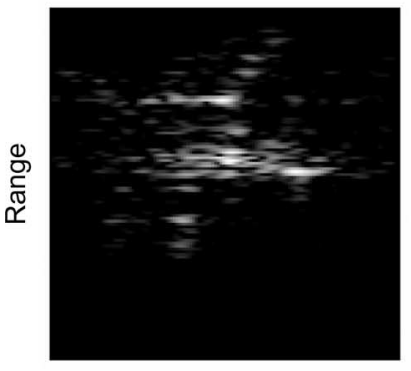

Cross-range

(c)

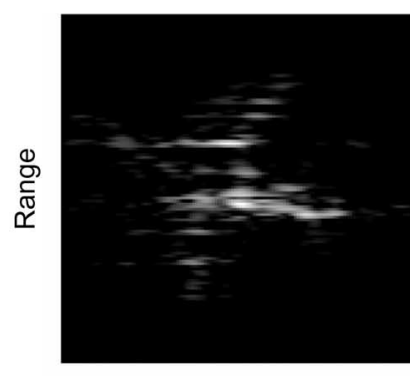

Cross-range

(b)

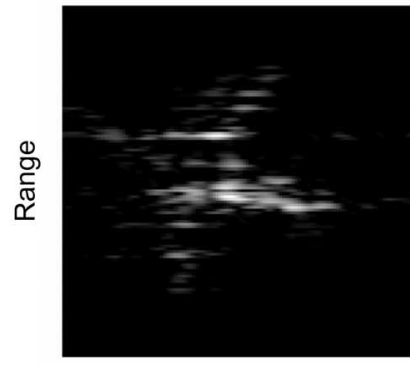

Cross-range

(d)

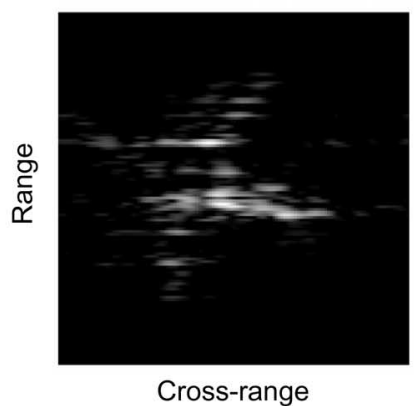

(e)

Fig. 6. ISAR images of a real YK-42 aircraft. (a) Unfocused, (b) focused by HOS, (c) focused by SOS, (d) focused by maximum image contrast, and (e) focused by minimum image entropy. 
TABLE I

IMAge QuALity MEASURES IN FIg. 3

\begin{tabular}{|c|c|c|c|c|c|}
\hline Fig. 3 & (a) & (b) & (c) & (d) & (e) \\
\hline IC & 1.38 & 1.76 & 1.70 & 1.77 & 1.71 \\
\hline IP & 215.67 & 509.24 & 476.30 & 561.55 & 550.76 \\
\hline IE & 7.55 & 6.40 & 6.41 & 6.24 & 6.23 \\
\hline
\end{tabular}

TABLE II

IMAGE QUALITY MEASURES IN Fig. 6

\begin{tabular}{|c|c|c|c|c|c|}
\hline Fig. 6 & (a) & (b) & (c) & (d) & (e) \\
\hline IC & 1.14 & 1.47 & 1.35 & 1.48 & 1.47 \\
\hline IP & 620.52 & 882.22 & 685.18 & 907.01 & 902.33 \\
\hline IE & 8.32 & 8.03 & 8.25 & 8.02 & 8.01 \\
\hline
\end{tabular}

The second ISAR data set was processed. The object was a YK-42 aircraft. The C-band radar transmitted a linear FM signal with a bandwidth of $400 \mathrm{MHz}$. The PRF was $400 \mathrm{~Hz}$. The aircraft was at the broadside position. Thirty-two return signals were used for ISAR imaging. The change of aspect angle was $0.3^{\circ}$. The two assumptions made for the signal model are valid. The ISAR images of YK-42 are shown in Fig. 6 where (a) is the unfocused image, (b) is the focused image with the HOS approach, (c) is the focused image with the SOS approach, (d) is the focused image with the maximum image contrast method, and (e) is the focused image with the minimum image entropy method. The focused images are satisfactory.

Image contrasts (IC) [5], image peak values (IP) [5], and image entropies (IE) [6] in the subplots of Figs. 3 and 6 are shown in Tables I and II, respectively. They indicate that the maximum image contrast produces the largest image contrast. HOS is slightly better in image contrast than the minimum image entropy. The image contrast of SOS is smaller than that of the minimum image entropy. With regard to the image peak value, the maximum image contrast generates the maximum image peak value. The image peak value of the HOS is smaller than that of the minimum image entropy but is larger than that of the SOS. For image entropy, the minimum image entropy provides the smallest image entropy. The image entropy obtained by HOS is less than that of SOS but is greater than that of the maximum image contrast.

\section{CONCLUSION}

An approach based on HOS has been proposed to carry out ISAR autofocus. Its advantages over conventional methods are to suppress additive noise, enhance SNR, and provide a low SNR threshold. The high-quality ISAR images have been produced by processing real data. Statistical tests show that the developed HOS approach generates a higher focused image at a given SNR level and has a SNR gain of $10 \mathrm{~dB}$ for the same focused quality compared with the SOS method when the SNR is less than $-5 \mathrm{~dB}$. In comparison with the maximum image contrast and the minimum image entropy methods, the developed approach has a numerical stability and a small computational load, although it has a small loss in image quality.

\section{ACKNOWLEDGMENT}

The authors would like to thank the associate editors and the reviewers for their comments to improve the quality of presentation.

\section{REFERENCES}

[1] D. A. Ausherman, A. Kozma, J. L. Walker, H. M. Jones, and E. C. Poggio, "Developments in radar imaging," IEEE Trans. Aerosp. Electron. Syst., vol. AES-20, no. 4, pp. 363-400, Jul. 1984.

[2] C. C. Chen and H. C. Andrews, "Target-motion-induced radar imaging," IEEE Trans. Aerosp. Electron. Syst., vol. AES-16, no. 1, pp. 2-14, Jan. 1980.

[3] B. D. Steinberg, "Microwave imaging of aircraft," Proc. IEEE, vol. 76, no. 12 , pp. $1578-1592$, Dec. 1988

[4] D. E. Wahl, P. H. Eichel, D. C. Ghiglia, and C. V. Jakowatz, Jr., "Phase gradient autofocus-A robust tool for high resolution SAR phase correction," IEEE Trans. Aerosp. Electron. Syst., vol. 30, no. 3, pp. 827-835, Jul. 1994.

[5] F. Berizzi and G. Corsini, "Autofocusing of inverse synthetic aperture radar images using contrast optimization," IEEE Trans. Aerosp. Electron. Syst., vol. 32, no. 3, pp. 1185-1191, Jul. 1996.

[6] X. Li, G. Liu, and J. Ni, "Autofocusing of ISAR images based on entropy minimization," IEEE Trans. Aerosp. Electron. Syst., vol. 35, no. 4, pp. 1240-1252, Oct. 1999.

[7] Z. She, R. E. Bogner, and D. A. Gray, "An eigenvector approach for ISAR motion compensation and imaging," in Proc. IEEE TENCON, Brisbane, Australia, 1997, pp. 659-662.

[8] C. L. Nikias and A. P. Petropulu, Higher-Order Spectra Analysis: A Nonlinear Signal Processing Framework. Englewood Cliffs, NJ: PrenticeHall, 1993.

[9] Z. She, D. A. Gray, and R. E. Bogner, "Autofocus for ISAR imaging," Signal Process., vol. 81, no. 2, pp. 275-291, 2001.

[10] J. M. Mendel, "Tutorial on higher-order statistics (spectra) in signal processing and system theory: Theoretical results and some applications," Proc. IEEE, vol. 79, no. 3, pp. 278-305, Mar. 1991.

[11] F. Berizzi, G. Corsini, and F. Gini, "HOS based algorithm for autofocusing of spotlight SAR images," Electron. Lett., vol. 33, no. 7, pp. 628-630, Mar. 1997.

[12] J. Wang and X. Liu, "SAR minimum-entropy autofocus using an adaptiveorder polynomial model," IEEE Geosci. Remote Sens. Lett., vol. 3, no. 4, pp. 512-516, Oct. 2006.

[13] J. Xu, Y. Peng, and X. Xia, "Parametric autofocus of SAR imaging-Inherent accuracy limitation and realization," IEEE Trans. Geosci. Remote Sens., vol. 42, no. 11, pp. 2397-2411, Nov. 2004. 